\title{
Exploring handedness asymmetries in the Simon effect
}

\author{
Julia C. Seibold ${ }^{1} \cdot$ Jing Chen $^{2} \cdot$ Robert W. Proctor ${ }^{1}$
}

Published online: 16 December 2015

(C) The Psychonomic Society, Inc. 2015

\begin{abstract}
Spatially corresponding stimulus-response pairings usually produce shorter reaction times (RTs) than do noncorresponding pairings, even when the spatial dimension of the stimulus is irrelevant to the task. This "Simon effect" for visual stimuli and manual responses is often larger for the stimulus location on the side to which the person's dominant hand is operating. The present study aimed at replicating and examining the nature of this asymmetry. To determine whether the Simon effect asymmetry is a function of the hand distinction or of conceptual spatial codes, performance with left and right manual key-presses was compared to that with vocal responses "left" and "right." Whether the asymmetric Simon effect pattern is restricted to spatial stimuli was tested by comparing effects obtained with left and right located squares to those found with the centered words Left and Right. The asymmetry was only replicated for the spatial stimulusmanual response Simon task, for which a second experiment showed similar results when the hands and response box were not visible during task performance. The analysis revealed a general dominant-hand RT advantage that similarly adds to both corresponding and non-corresponding trials and is rather independent from the Simon effect. This advantage yields an apparent asymmetry when the data are analyzed as a function of correspondence for each stimulus location because the corresponding and non-corresponding RTs that are compared come from different hands.
\end{abstract}

Robert W. Proctor

proctor@psych.purdue.edu

1 Department of Psychological Sciences, Purdue University, 703 Third Street, West Lafayette, IN 47907-2081, USA

2 Department of Psychology, New Mexico State University, Las Cruces, NM, USA
Keywords Attention · Perception and action · Response selection - Spatial compatibility · Stimulus-response compatibility

\section{Introduction}

For two-choice reaction tasks in which a left or right response is made to a stimulus presented randomly in a left or right location, a compatible mapping of stimulus and response locations produces shorter reaction times (RTs) than does an incompatible mapping. This RT difference is called a stimulus-response (S-R) compatibility (SRC) effect (Fitts \& Deininger, 1954; for a review see Proctor \& Vu, 2006). In most theoretical accounts, the SRC effect is attributed at least in part to activation of the spatially corresponding response that occurs as a consequence of the similarity, or overlap, of the stimulus and response dimensions (De Jong, Liang, \& Lauber, 1994; Kornblum, Hasbroucq, \& Osman, 1990).

A similar yet smaller SRC effect occurs when stimulus location is irrelevant to the task. This latter effect was first detected by Simon and Small (1969), who had participants respond with a left or right key-press to the pitch of a tone presented to the left or right ear. Although only tone pitch was relevant, responses were faster when the stimulus and response locations corresponded than when they did not. This correspondence effect, named the Simon effect (Hedge \& Marsh, 1975; Simon, 1990), has been studied extensively ever since, more often with visual than auditory stimuli (e.g., Töbel, Hübner, \& Stürmer, 2014).

Researchers often collapse across stimulus and response locations in studies of the Simon effect, examining the difference in RT for non-corresponding and corresponding trials. However, studies reported within the past 10 years have questioned this practice, noting that the Simon effect is often asymmetric across the left and right positions (Rubichi \& 
Nicoletti, 2006; Spironelli, Tagliabue, \& Umiltà, 2009; Tagliabue et al., 2007). Those authors have also argued that accounts of the Simon effect that ignore the asymmetry are incomplete and have proposed that the asymmetry reflects stronger coding of stimulus location in the visual hemifield corresponding to the dominant hand, as a consequence of an attentional bias. In the present study, we consider these issues in the context of versions of Simon tasks that differ in spatial versus verbal stimulus modes and spatial versus vocal response modes.

\section{The Simon effect}

The Simon effect has some central characteristics (Lu \& Proctor, 1995). For one, it does not depend on absolute stimulus and effector locations but on the relative positions of the stimuli and responses, as shown by Wallace (1971). In his study, participants performed a Simon task while holding their hands crossed, with the right hand operating the left response key and the left hand the right key. Similar to when the hands are in uncrossed natural positions, responses were faster when stimulus and response locations corresponded than when they did not. The anatomical feature of the effector (right or left hand) did not have an influence on the Simon effect. In agreement, Umiltà and Nicoletti (1985) obtained similar-sized Simon effects when left and right stimuli and left and right response keys were both located to the left or to the right of body midline. Many studies like those of Wallace and of Umiltà and Nicoletti have also found evidence in Simon and SRC tasks that spatial stimulus and response information is coded independent of effector and body position (e.g., Nicoletti, Anzola, Luppino, Rizzolatti, \& Umiltà, 1982).

The most common explanation of the Simon effect is a dualroute account (De Jong et al., 1994; Kornblum et al., 1990). The response that corresponds to the stimulus location is activated via a direct, automatic response-activation route. The second route is indirect and intentional, as it activates a response to the stimulus based on the instructed task requirements. On a corresponding trial both routes activate the same response, but on a non-corresponding trial different responses are activated, resulting in competing response tendencies. Consistent with the view that the Simon effect reflects automatic activation of the corresponding response, it typically decreases across the RT distribution (e.g., De Jong et al., 1994).

Other explanations place emphasis on the nature of the spatial codes that underlie the Simon effect. According to the referential coding account offered by Hommel (1993b), the spatial stimulus codes are formed in relation to reference frames in the task environment. Alternatively, Nicoletti and Umiltà's (1994) attention shift account attributes the spatial stimulus codes that are produced to shifts of attentional focus from one location (presumably fixation) to another (the position of the relevant stimulus). Further, the theory of event coding (Hommel, Müsseler, Aschersleben, \& Prinz, 2001) assumes that actions are coded in terms of their perceivable effects. The response-key mapping of a Simon task, which requires distinguishing left- and right-located key-presses, therefore entails that location is not really irrelevant (see also Ansorge \& Wühr, 2004; Yamaguchi \& Proctor, 2012).

Regardless of the exact explanation of the Simon effect, it has turned out to be a very useful tool to study the processing of irrelevant spatial information in a straightforward and isolated way. It allows for controlling and manipulating the relations between stimulus and response features, enabling the amount of cognitive conflict to be measured (Hommel, 2011). Hence, the Simon effect has become an essential instrument for investigating perceptual, attentional, and action planning processes, as well as cognitive control mechanisms.

\section{The Simon effect asymmetry}

Based on a review of several articles in which Simon effect data were reported as a function of stimulus and response locations, Rubichi and Nicoletti noted that the Simon effect often was larger for the right visual stimulus than for the left one (e.g., Nicoletti, Umiltà, \& Mapelli, 1992; Proctor \& Wang, 1997; Valle-Inclán, Sohn, \& Redondo, 2008; Wallace, 1971). Rubichi and Nicoletti (2006) supposed that the likely reason for this Simon effect asymmetry was that most participants were right-handed. They cited a study of Rabbitt (1978) showing that responses of the dominant right hand were faster for tasks containing a choice between hands, which he interpreted as due to an attentional bias. Based on Rabbitt's interpretation, and Nicoletti and Umiltà's (1994) attention shift account of the Simon effect, Rubichi and Nicoletti (2006) proposed that "spatial attention shifts are biased toward the part of the space where the dominant effector is operating" (p. 1067), producing stronger spatial codes for the stimulus to that side.

To test this claim that a dominant-hand centered attentional bias produces the Simon effect asymmetry, Rubichi and Nicoletti (2006) aimed to replicate the RT pattern in a visual Simon task with three groups of participants: left-handed, right-handed, and ambidextrous. Red and green squares were presented in random order to the left or right of a central fixation cross on a display screen, to which participants responded with a left or right key-press. The task was performed with uncrossed hands in Experiment 1, with correspondence (the difference between non-corresponding and corresponding responses) for each stimulus location analyzed. The resulting Simon effect interacted with stimulus location and handedness, as indicated by a significant interaction of stimulus location $\times$ correspondence $\times$ handedness. As expected, for right-handed participants the Simon effect was larger 
for stimuli presented in the right location than for those presented in the left location. Left-handed participants showed an opposite pattern, and ambidextrous participants showed no asymmetry. For Experiment 2, in which participants responded with the hands crossed, the results showed reversed asymmetries: the left-handed group evidenced a larger Simon effect for stimuli in the right location, whereas the righthanded group showed a larger Simon effect for stimuli in the left location. Consequently, Rubichi and Nicoletti concluded that the asymmetry is influenced by the location of the dominant hand and not by the hands' natural sides of operation (left of body midline for the left hand and right of midline for the right hand).

For Rubichi and Nicoletti's (2006) Experiment 1, post-hoc $t$-tests of the stimulus location $\times$ correspondence $\times$ handedness interaction revealed a significant difference between the two stimulus locations for the corresponding trials only. The authors interpreted this result as evidence against an unspecific motoric or cognitive preparation advantage for the dominant hand, because such an advantage should also affect the noncorresponding trials. For Experiment 2, analysis of the RT distribution for corresponding trials revealed that the dominant-hand advantage increased as RT increased, which Rubichi and Nicoletti interpreted against an execution advantage for the dominant hand (which should be most evident for fast responses) and consistent with an attention shift that requires time to occur. They concluded, "There is evidence ... of an attentional bias centered on the dominant hand" (p. 1067), and this bias is due to "a larger cortical representation of the dominant hand" (p. 1068).

Tagliabue et al. (2007) reported similar experiments on the Simon effect asymmetry. They also found that for righthanded participants the Simon effect was larger for the right stimulus location than for the left one, whereas for left-handed participants the opposite relation held. Tagliabue et al. made stronger claims than Rubichi and Nicoletti, concluding that their study "provides a strong argument against collapsing data from the two sides to measure the Simon effect" (p. 50) and "collapsing left and right RTs into the single factor of 'correspondence' should be avoided" (p. 60). These statements imply that conclusions based on the overall Simon effect without considering the asymmetry may need to be reevaluated.

Although Tagliabue et al. (2007) interpreted their results as agreeing with an attention shift account and against a referential coding account, they also noted that the existing theories to explain the Simon effect were lacking an explanation for the asymmetry. In a later study, Spironelli et al. (2009) developed a connectionist model for righthanded participants to explain the asymmetry. Based on neural evidence that indicates that the visuo-spatial attention system is asymmetric in right-handers, Spironelli et al. modeled two layers to mimic the right superior parietal lobule being activated by left- and right-lateralized stimuli, and the left superior parietal lobule only by right-lateralized stimuli. This additional attention mechanism allowed the model to generate the asymmetric Simon effect pattern reported by Rubichi and Nicoletti (2006), and Tagliabue et al.

\section{Present study}

Before requiring researchers to report the Simon effect separately for the individual stimulus (or response) locations and going further in developing models of the Simon effect asymmetry, research needs to determine whether handedness contributes directly or indirectly to the Simon effect, as well as whether the contribution is restricted to task variations in which handedness would presumably play a role. Apart from testing participants of different handedness, the assumption of handedness as responsible for the Simon effect asymmetry can be tested by comparing performance with manual left and right key-press responses with the spoken responses "left" and "right" (e.g., Wühr, 2006). If handedness is restricted to manual responses, as the term implies, and an attention bias toward the side of the dominant hand is the source of the Simon effect asymmetry, then the asymmetry should not occur for the vocal responses. Likewise, the Simon effect with spatial stimuli can be compared to that with the centrally presented words Left and Right, for which the location information is conceptual. If handedness involves a bias of visual attention to the right side, no asymmetry should be evident with the centered stimuli of the location-word version of the Simon task.

The manipulations of the two stimulus modes and two response modes yield four Simon task conditions, which we tested in Experiment 1: (a) spatial stimuli and manual responses (spatial-manual condition); (b) word stimuli and manual responses (verbal-manual condition); (c) spatial stimuli and spoken responses (spatial-vocal condition); and (d) word stimuli and spoken responses (verbal-vocal condition). Several outcomes in terms of the Simon effect asymmetry are possible for these conditions. First, a replication of the asymmetric Simon effect as a function of stimulus location and correspondence should be obtained with manual responses to spatial stimuli. Second, if this asymmetry is evident with spoken responses to spatial stimuli, then physical stimulus location is likely to be the main source of the asymmetry. Third, if the asymmetry is found with manual responses to word stimuli, the implication would be that handedness of the participants produces the asymmetries, but independent of a need to direct attention to the left or right hemifield. Fourth, regardless of which of the prior result patterns is found, the condition with verbal stimuli and vocal responses (which does not include any physical spatial task feature) should not produce the asymmetric Simon effect. The presence of an asymmetry in that condition would imply that it is 
more conceptual than perceptual-motor in nature. In contrast, if an asymmetric Simon effect is evident only in the replication condition, this result would suggest that the phenomenon is based on an interplay of spatial-motoric associations.

In Experiment 1 the interaction pattern indicative of the Simon effect asymmetry was evident only in the spatialmanual condition, with just the right-handed participants showing a significant asymmetry. Therefore, in Experiment 2 we tested only right-handed participants to determine whether vision of the hands and response apparatus was necessary to obtain the asymmetry. In both experiments we also explored whether the asymmetry is truly an inseparable part of the Simon effect, as claimed by Rubichi and Nicoletti (2006), Tagliabue et al. (2007), and Spironelli et al. (2009) in postulating that a dominant-hand centered bias influences the attention shifts between fixation and stimulus. There is room for doubt in this regard as the asymmetry was mainly found for corresponding trials between stimulus locations in Rubichi and Nicoletti's (2006) Experiment 1, though not in their Experiment 2. Further, one could also think of a different outcome. Namely, the anatomical factor handedness can produce a bias towards one side that should increase or attenuate the Simon effect as a whole (Hommel, 1993a), instead of distorting it in the corresponding conditions only.

Before describing the experiments, we emphasize the following general points about our methods of data analysis. We analyze the results with the factors of stimulus location and response location rather than stimulus location and correspondence, as Rubichi and Nicoletti (2006) did. The two analyses yield the same $F$ ratios, just rearranged in terms of their assignment to effects (see Table 1), but Tagliabue et al. (2007) noted that the stimulus and response locations version leads to a more straightforward interpretation of the data than does the stimulus location $\times$ correspondence version. The reason is that the latter version confounds the hand distinction with the Simon effect: For the stimulus on the side of the dominant hand, corresponding trials with the dominant hand are compared to non-corresponding trials with the non-

Table 1 Comparison of two different analysis of variance (ANOVA) designs for evaluating the Simon effect asymmetry, employing the factors of stimulus location (StL), response location (ReL), correspondence (C), and handedness $(\mathrm{H})$

\begin{tabular}{lll}
\hline & Tagliabue-ANOVA & Rubichi-ANOVA \\
\hline 1 & $\mathrm{StL}$ & $\mathrm{StL}$ \\
2 & $\mathrm{ReL}$ & $\mathrm{StL} \times \mathrm{C}$ \\
3 & $\mathrm{StL} \times \mathrm{H}$ & $\mathrm{StL} \times \mathrm{H}$ \\
4 & $\mathrm{ReL} \times \mathrm{H}$ & $\mathrm{StL} \times \mathrm{C} \times \mathrm{H}$ \\
5 & $\mathrm{StL} \times \mathrm{ReL}$ & $\mathrm{C}$ \\
6 & $\mathrm{StL} \times \mathrm{ReL} \times \mathrm{H}$ & $\mathrm{C} \times \mathrm{H}$ \\
\hline
\end{tabular}

Note: The terms on each row yield the same $F$ ratios dominant hand, whereas for the stimulus on the opposite side, corresponding trials with the non-dominant hand are compared to non-corresponding trials with the dominant hand.

This relation implies that the interaction of stimulus location $\times$ correspondence represents only a response location main effect, as Tagliabue et al. (2007) show. Hence, in Rubichi and Nicoletti's (2006) analysis an RT advantage of the dominant hand (the factor of response location within each handedness group) is represented by the interaction of stimulus location $\times$ correspondence $\times$ handedness. Consequently, the terms "dominant-hand advantage" and "Rubichi and Nicoletti's Simon effect asymmetry" can be used interchangeably because they refer to the same effect. Throughout our study, which uses the ANOVA design with the factors stimulus location, response location, and handedness, the Simon effect asymmetries reported by Rubichi and Nicoletti can be seen in the interaction of response location $\times$ handedness. To clarify the relation between the Simon effect and this dominant-hand advantage, the time courses of these two effects will be examined across the RT distribution, as Rubichi and Nicoletti did in their Experiment 2.

\section{Experiment 1}

\section{Method}

Participants Twenty-four left-handed (16 female) and 24 right-handed (15 female) undergraduate students, enrolled in Introductory Psychology classes at Purdue University, took part for course credits. They were invited based on their responses to a handedness question on a preliminary questionnaire, although they were not aware of the reason why they were invited. The Edinburgh handedness questionnaire (Oldfield, 1971) was filled out at the start of the experiment. The handedness questionnaire categorizes participants with values from -100 to -40 with a mean score of $-82.19(S D=$ $19.03)$, and from 40 to 100 with a mean score of 91.51 ( $S D=$ 14.43) as left- and right-handed, respectively. A comparison of absolute values for the two groups showed a tendency toward stronger dominance for the right-handed participants, $t(47)=1.89, p=0.065$. The mean age of the left-handed participants was 18.38 years $(S D=0.58)$, and that of the right-handed participants was 18.50 years $(S D=0.78)$.

Apparatus and stimuli The experiment was conducted on a DELL Optiplex 745 personal computer with a DELL 19-in. LCD color monitor, and controlled by E-Prime Software (Version 2.0). The monitor was viewed at a distance of approximately $55 \mathrm{~cm}$. The experiment consisted of two parts, one with manual responses (left and right key-presses) and one with vocal responses (spoken words "left" and "right"). Within these parts there was one block of spatial stimuli and a 
block of location-word stimuli. The spatial stimuli were red and green rectangles of $1.15^{\circ} \times 0.94^{\circ}$ visual angle, presented $4.50^{\circ}$ visual angle to the left or right of a centered fixation cross $\left(0.83^{\circ}\right)$. The location-word stimuli were the words Left and $\operatorname{Right}\left(3.33^{\circ} \times 0.94^{\circ}\right.$ and $4.17^{\circ} \times 0.94^{\circ}$ visual angle $)$ with the first letter capitalized and printed in red or green, presented centrally $2.40^{\circ}$ above the fixation cross. All stimuli were displayed on a white background. Manual responses were made on the leftmost and rightmost keys out of five keys on a Serial Response Box (Psychology Software Tools, Inc., Pittsburgh, PA, USA) with the left and right index fingers, respectively. In contrast, in the vocal response part of the experiment, the spoken responses were detected by an ATR20 cardioid microphone (Audio-Technica) plugged into the voice key of the Response Box, followed by an identifying key press made by the experimenter (this is a common practice of recording auditory responses, e.g., Wühr, 2006). The mapping of colors to response sides/words and the sequence of the manual and verbal parts of the experiment were counterbalanced between participants. The same applies to the sequence of spatial and location-word blocks. Yet, the latter order stayed the same within a participant for the manual and the verbal parts of the experiment.

Each quarter of the experiment contained 12 practice trials, followed by 120 experimental trials with stimulus colors and locations randomly intermixed. There were 60 trials in which the stimulus information (spatial or verbal) and response location (manual or vocal) corresponded, and another 60 trials for which they did not correspond. Each trial started with a 1,000ms presentation of the fixation cross, followed by a $100-\mathrm{ms}$ warning tone $(400 \mathrm{~Hz})$. After that, the stimulus appeared and stayed on the screen until a response was registered. For the vocal response conditions, the experimenter entered the response identity on a separate keyboard by pressing the corresponding left or right arrow key. For the manual response conditions, the experimenter similarly pressed a left or right arrow key, but the participant's key-press was used to identify their response. Following the experimenter's action, a 500-ms feedback screen appeared that showed just the fixation cross for correct responses or the word "Error" at $2.40^{\circ}$ below the fixation cross for incorrect responses.

Procedure After giving their informed consent, participants filled out the Edinburgh handedness inventory (Oldfield, 1971) and then were instructed about the Simon task. They were told to respond as fast and accurately as possible to the color of each stimulus by pressing the assigned response key or saying the assigned response ("left" or "right"). Half of the participants were to press the left key (or say "left") for red stimuli, and the right key (or say "right") for green stimuli, whereas the other half had the opposite assignment. The assignment was fixed for each participant throughout the whole experiment.

\section{Results}

Following Rubichi and Nicoletti (2006), only trials with RTs in the range of 100-900 ms were included in the analysis. The overall error rate within these $96.1 \%$ of the trials was $2.9 \%$, and the RT of the correct trials was $489 \mathrm{~ms}$. As noted, we report the data analysis using the ANOVA design of Tagliabue et al. (2007), which contains the factors stimulus location, response location, and handedness, as well as stimulus mode and response mode. Handedness was the only between-subject factor. All significant effects are reported.

Reaction time The mean RT data are shown in Fig. 1. The ANOVA showed a main effect of response location, $F(1,46)=8.50, p=.005, \eta^{2}=.16$, indicating a shorter RT for the right response $(M=489 \mathrm{~ms})$ than the left response $(M=495 \mathrm{~ms})$. There was also a main effect of response mode, $F(1,46)=187.68, p<.001, \eta^{2}=.80$, with RTs longer for the vocal mode than for the manual mode $(\Delta M=$ $115 \mathrm{~ms})$. Response mode interacted with stimulus mode, $F(1$, 46) $=7.97, p=.007, \eta^{2}=.15$, signifying that the difference was larger with spatial stimuli $(\Delta M=126 \mathrm{~ms})$ than with location-word stimuli $(\Delta M=103 \mathrm{~ms})$. The main effect of stimulus location was not significant, but location interacted with handedness, $F(1,46)=8.16, p=.006, \eta^{2}=.15$ : Righthanded participants showed a small advantage $(5 \mathrm{~ms})$ for the right stimulus location, whereas left-handed participants showed a slight opposite tendency $(-2 \mathrm{~ms})$.

The stimulus location $\times$ response location interaction was also significant, $F(1,46)=97.62, p<.001, \eta^{2}=.68$, signifying a $20-\mathrm{ms}$ Simon effect. These two factors entered into an interaction with stimulus mode, $F(1,46)=13.00$, $p<.001, \eta^{2}=.22$, as well as a 4-way interaction of those variables with response mode, $F(1,46)=11.81, p<.001$, $\eta^{2}=.20$, but not a 5 -way interaction with handedness, $F<1.0$. This 4-way interaction paired with no 5 -way interaction means that the Simon effect varied across the conditions created by the pairings of stimulus modes and response modes, and, importantly, that these differences were not influenced by handedness (see Fig. 1).

The interaction term that would be indicative of the asymmetry reported by Rubichi and Nicoletti (2006) is the 4-way interaction of stimulus mode $\times$ response mode $\times$ response location $\times$ handedness. This interaction was also not significant, $F(1,46)=2.72, p=.106, \eta^{2}=.06$, but because the asymmetry was expected to be replicated in the spatialmanual condition and possibly not in the other three conditions, we conducted a similar ANOVA with the four conditions as a single factor partitioned into two orthogonal contrasts. The contrast comparing the spatial-manual condition to the other three conditions showed a significant interaction of condition, response location, and handedness, $F(1,46)=6.10$, $p=.017, \eta^{2}=.11$, whereas the contrast comparing the other 
(a) Spatial-Manual

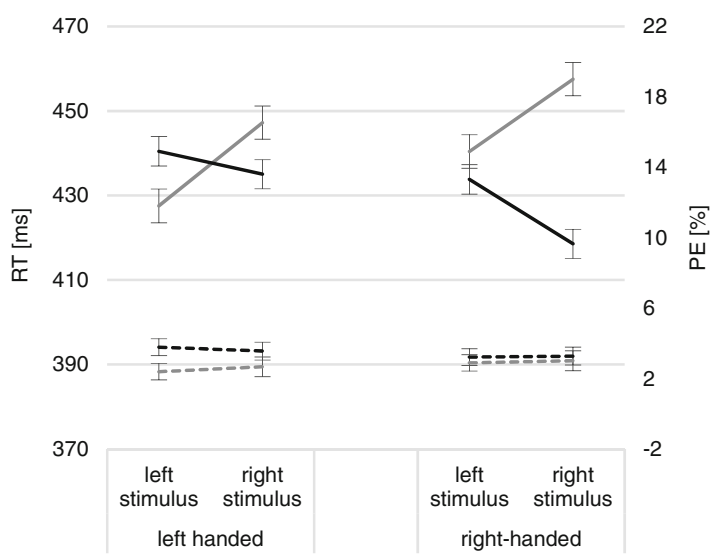

(c) Spatial-Vocal

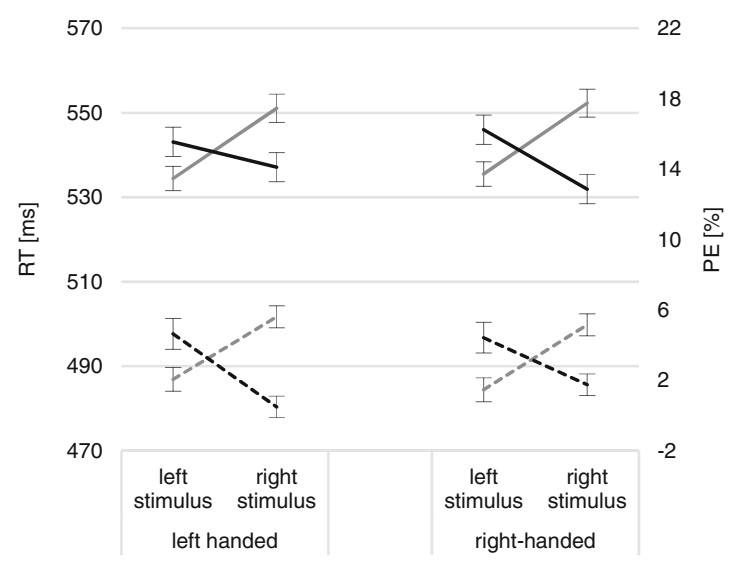

left response RT

right response $\mathrm{RT}$

Fig. 1 Mean reaction time (RT) and percent error (PE) values and Cousineau-Morey confidence intervals (controlling for betweensubjects variability; Morey, 2008), as functions of stimulus location (left stimulus vs. right stimulus), response location (left response vs. right

three conditions did not, $F<1.0$. To clarify further whether this result pattern indicates that the Simon effect asymmetry was replicated in the spatial-manual condition but not evident in each of the other conditions, we conducted separate ANOVAs for the four conditions (see Table 2 for ANOVAs).

Spatial-manual condition For the spatial-manual condition, there was a main effect of response location, due to RT being $11 \mathrm{~ms}$ shorter with the right response than the left response. More importantly, the Simon effect asymmetry reported by Rubichi and Nicoletti (2006) was found, which is indicated by the critical interaction of response location $\times$ handedness: right-handed participants reacted faster with the dominant hand than with the non-dominant left hand $(\Delta M=23$ ms, $p<.001)$, whereas left-handed participants showed no dominant-hand advantage $(\Delta M=0.5 \mathrm{~ms}, p=.934)$. Note that (b) Verbal-Manual

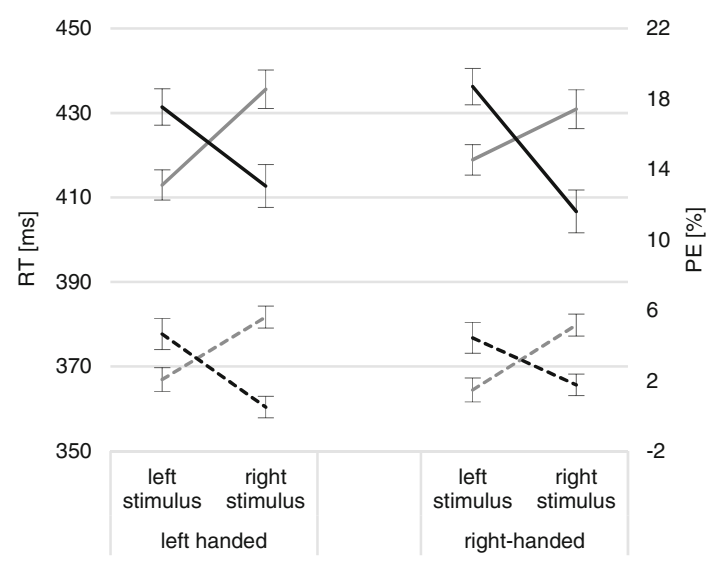

(d) Verbal-Vocal

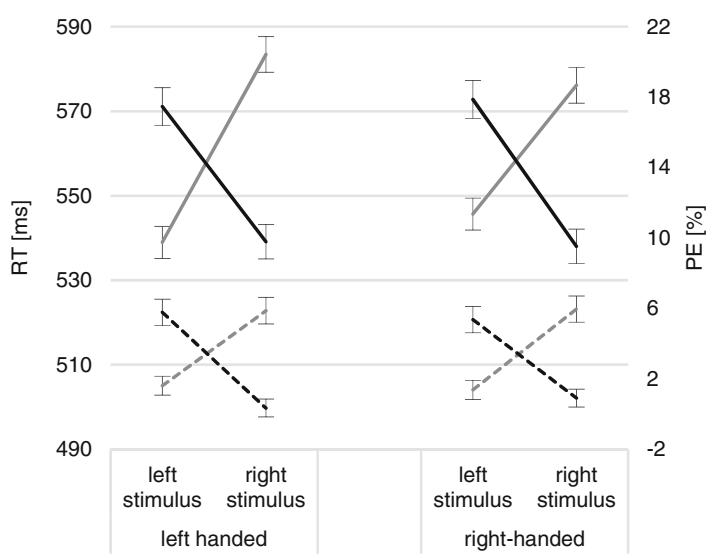

left response $\mathrm{PE}$

right response $\mathrm{PE}$

response), and handedness (left-handedness vs. right-handedness) for the (a) spatial-manual condition, (b) verbal-manual condition, (c) spatial-vocal condition, and (d) verbal-vocal condition

although this interaction is that of stimulus location, correspondence, and handedness in the analysis reported by Rubichi and Nicoletti (see Table 1), it does not involve the Simon effect, but only a difference in RTs for the two responses (one with the left hand and one with the right hand).

Planned orthogonal comparisons like those reported by Tagliabue et al. (2007) showed a difference in Simon effect for the left and right stimuli only for right-handed participants (see also Fig. 1a): the Simon effect for right stimuli was large ( $\Delta M=42 \mathrm{~ms}, p<.001$ ), but the Simon effect for left stimuli was not present $(\Delta M=-5 \mathrm{~ms}, p=.323)$. In contrast, the lefthanded participants revealed similar Simon effects for both left and right stimuli ( $\Delta M=13 \mathrm{~ms}, \Delta M=12 \mathrm{~ms}, p s=.055$ and .065 , respectively), rather than the complete reversal obtained by Rubichi and Nicoletti. The lack of reversal may be due to handedness being less pronounced for the left-handed 
Table 2 Experiment 1: Reaction time (RT) and percent error (PE) ANOVA results of the four stimulus-response conditions, with the factors of stimulus location (StL), response location (ReL), and handedness $(\mathrm{H})$

\begin{tabular}{|c|c|c|c|c|c|c|c|c|}
\hline & \multicolumn{2}{|c|}{ Spatial-manual } & \multicolumn{2}{|c|}{ Verbal-manual } & \multicolumn{2}{|c|}{ Spatial-vocal } & \multicolumn{2}{|c|}{ Verbal-vocal } \\
\hline & $F$ & $p$ & $F$ & $p$ & $F$ & $p$ & $F$ & $p$ \\
\hline \multicolumn{9}{|l|}{$R T$} \\
\hline StL & 2.58 & 0.115 & 2.22 & 0.143 & 2.13 & 0.151 & 0.53 & 0.470 \\
\hline $\mathrm{ReL}$ & 13.12 & $0.001 * *$ & 0.61 & 0.438 & 1.51 & 0.225 & 2.42 & 0.126 \\
\hline $\mathrm{StL} \times \mathrm{H}$ & 1.51 & 0.230 & 5.55 & $0.023 *$ & 0.76 & 0.387 & 2.24 & 0.142 \\
\hline $\mathrm{ReL} \times \mathrm{H}$ & 13.98 & $0.001 * *$ & 0.28 & 0.867 & 0.13 & 0.716 & 0.01 & 0.937 \\
\hline $\mathrm{StL} \times \mathrm{ReL}$ & 17.73 & $0.001 * *$ & 20.60 & $0.001 * *$ & 25.15 & $0.001 * *$ & 99.14 & $0.001 * *$ \\
\hline $\mathrm{StL} \times \mathrm{ReL} \times \mathrm{H}$ & 0.28 & 0.600 & 0.00 & 0.992 & 0.60 & 0.442 & 0.63 & 0.432 \\
\hline \multicolumn{9}{|l|}{$P E$} \\
\hline $\mathrm{StL}$ & 0.02 & 0.902 & 0.07 & 0.795 & 0.00 & 0.968 & 0.30 & 0.589 \\
\hline ReL & 3.68 & 0.061 & 2.77 & 0.103 & 1.07 & 0.306 & 1.93 & 0.171 \\
\hline $\mathrm{StL} \times \mathrm{H}$ & 0.01 & 0.945 & 1.06 & 0.308 & 0.64 & 0.427 & 0.48 & 0.493 \\
\hline $\mathrm{ReL} \times \mathrm{H}$ & 1.26 & 0.268 & 1.38 & 0.246 & 1.79 & 0.188 & 0.03 & 0.869 \\
\hline $\mathrm{StL} \times \mathrm{ReL}$ & 0.14 & 0.708 & 19.26 & $0.001 * *$ & 2.30 & 0.136 & 52.72 & $0.001 * *$ \\
\hline $\mathrm{StL} \times \mathrm{ReL} \times \mathrm{H}$ & 0.08 & 0.784 & 0.18 & 0.678 & 0.48 & 0.494 & 0.07 & 0.800 \\
\hline
\end{tabular}

Note: $* * p<.001 ; * p<.05$

participants in our study. We also point out the absence of a 3way interaction of stimulus location and response location with handedness, $F<1.0$, in the main analysis of the spatialmanual condition. This absence means that the size of the overall spatial-manual Simon effect was not influenced by handedness and the asymmetry as a function of stimulus location within the Simon effect of the right-handed group.

Again following Rubichi and Nicoletti (2006), corresponding and non-corresponding trials to left and right stimuli were also compared separately within each handedness group. Deviating from the findings of Rubichi and Nicoletti's Experiment 1 (but not their Experiment 2, in which the hands were crossed), which showed a significant difference between stimulus locations only for corresponding trials, posthoc Bonferroni-corrected $t$-tests revealed differences between both corresponding ( $\Delta M=23 \mathrm{~ms}$ ) and non-corresponding trials $(\Delta M=24 \mathrm{~ms})$ for right-handed participants, $t s(23)=$ 3.60 and 3.83, $p s<.001$. For left-handed participants, neither corresponding nor non-corresponding trials differed between stimulus locations, $t s(23)=1.44$, and $.36, p s>.160$.

\section{Verbal-manual, spatial-vocal, and verbal-vocal conditions}

The verbal-manual condition showed an interaction of stimulus location $\times$ handedness (see Fig. 1b). However, neither it nor the other two conditions showed the crucial response location $\times$ handedness interaction indicative of Simon effect asymmetries of the type found for the spatialmanual condition in our study (see Fig. 1) and that of Rubichi and Nicoletti (2006).
Percent error The mean percent error (PE) data are plotted in Fig. 1a-d. A main effect of stimulus mode was found, $F(1,46)=5.46, p=.024, \eta^{2}=.11$, and stimulus mode interacted with response mode, $F(1,46)=6.68, p=.013$, $\eta^{2}=.13$. Pairwise comparisons showed that $1.0 \%$ fewer errors were made in the spatial-vocal condition than in the three other conditions, $p s<.044$, whereas there was no difference in the PEs of the other three conditions.

The ANOVA also revealed a significant stimulus location $\times$ response location interaction (i.e., Simon effect), $F(1,46)=$ $43.49, p<.001, \eta^{2}=.49$, with a difference in PE between noncorresponding and corresponding trials of $2.2 \%$. Stimulus mode interacted with stimulus location and response location, $F(1,46)=35.84, p<.001, \eta^{2}=.44$. Only in the conditions using verbal stimuli was a significant PE Simon effect found. In the post-hoc pairwise comparisons, the manual responses to verbal and spatial stimuli produced similar PEs $(p=.738)$, whereas for vocal responses the verbal stimuli produced $1.3 \%$ more errors than did the spatial stimuli $(p<.001)$, confirming the trends in the RT data.

Follow-up ANOVAs, conducted for each condition to complement those reported for the RT data, showed the following (see Table 2): For the spatial-manual condition, there were no significant effects, although the main effect of response location was numerically present, $F(1,46)=3.68, p=.061$, $\eta^{2}=.07$. Planned comparisons showed a slight tendency for a Simon effect for the left stimulus location, $t(47)=1.80$, $p=.078$, but the critical response location $\times$ handedness interaction was not significant. Similar ANOVAs revealed significant correspondence effects in the verbal-manual (3.6\%) 
and verbal-vocal (4.6\%) conditions, but not in the spatialvocal condition, and no Simon effect asymmetries.

The PE data for the verbal-manual condition showed a pattern of interaction of stimulus location $\times$ handedness that was opposite that of the RT data, which could indicate a speed-accuracy trade-off. In agreement with this possibility, when calculating the ANOVA with an inverse efficiency score $\left(\mathrm{IES}=\frac{\mathrm{RT}}{1-\mathrm{PE}}\right.$; Townsend \& Ashby, 1978) as the dependent variable, the interaction disappeared, $F(1,46)=1.27$, $p=.289, \eta^{2}=.02$, leaving only the strong main effect of correspondence.

Distribution analysis An RT distribution analysis is typically performed by doing the following for each participant (De Jong et al., 1994): correct RTs for corresponding and noncorresponding trials are rank ordered separately and divided into several bins, in the present case five (as in Rubichi \& Nicoletti (2006)'s Experiment 2). The Simon effect is calculated for each bin by subtracting the bin mean for corresponding trials from that for non-corresponding trials. The individual distribution functions are averaged to produce a group function, a process known as Vincentizing (Ratcliff, 1979).

The Simon effect for physical stimulus and response locations usually shows a decreasing RT function (e.g., De Jong et al., 1994; see Proctor, Miles, \& Baroni, 2011, for a review). These functions are typically presumed to reflect rapid activation and dissipation of the corresponding response. A distribution profile for the dominant-hand advantage can be calculated similarly by creating quintiles for each participant based on dominant and non-dominant hand responses (Rubichi \& Nicoletti, 2006). Then, the bin mean of the dominant hand response is subtracted from the bin mean of the nondominant hand response. PE within each bin can be analyzed in a similar manner, yielding a PE Simon effect function across the RT distribution (Ridderinkhof, 2002; Töbel et al., 2014; Xiong \& Proctor; 2015).

For the spatial-manual condition, ANOVAs were performed on the RT and PE data. Two separate ANOVAs were calculated for the Simon effect and the dominant-hand advantage, respectively, each containing the factors bin and handedness. This makes about 12 observations per bin cell for each participant, minus erroneous trials in RTs. In contrast to Rubichi and Nicoletti (2006), both corresponding and noncorresponding trials were considered in the analysis, as we found differences between stimulus locations also in noncorresponding trials. The RT data showed significant main effects of bin and Simon effect, as well as the critical interaction of both variables, $F(1.8,84.5)=21.05, p<.001, \eta^{2}=.31$. This interaction reflects a decreasing Simon effect function (see Fig. 2a), for which the Simon effect was present in the first to fourth bins $(p s<.05)$ but non-significant in the fifth bin $(p=.270)$. The 3-way interaction with handedness was not significant $(F<1.0)$. The PE ANOVA for the spatial-manual condition also showed a bin main effect and interaction of bin with the Simon effect, $F(4,184)=4.99, p=.001, \eta^{2}=.10$, with the Simon effect being mainly in the first bin, as other studies have found (e.g., Töbel et al., 2014). Again there was no interaction of the Simon effect with handedness $(F<1.0)$. The plotted RT data for the dominant-hand advantage in the spatial-manual condition showed a tendency to increase (see Fig. 2a), but the ANOVA of that advantage did not show the increase to be significant after Greenhouse-Geisser correction $(F<1.0)$, and did not interact with handedness, $F(1.5,69.8)=$ $2.35, p=.116$ ). No terms were significant in the PE ANOVA either.

Similar ANOVAs were performed for the three other experimental conditions (see Fig. 2c-d). The distribution of the RT Simon effect showed an increasing pattern in the verbal-manual condition, $F(1.5,67.6)=26.94, p<.001$, $\eta^{2}=.37$, and the verbal-vocal condition, $F(1.8,80.8)=24.04$, $\left.p<.001, \eta^{2}=.34\right)$, but no significant change in the PE Simon effect across bins. The spatial-vocal condition differed in this regard, showing no difference in the RT Simon effect across bins, but an interaction of bin, Simon effect, and handedness in PE, $\left.F(3.4,154.2)=3.27, p=.019, \eta^{2}=.07\right)$ : the only significant Simon effect in PE was in the fifth bin of the right-handed participants $(p=.002)$, for which the effect was $3.7 \%$. Besides the bin main effect in RT of the verbal-vocal condition, no effect was significant in RT or PE.

\section{Discussion}

The results of the spatial-manual condition replicated the main patterns reported by Rubichi and Nicoletti (2006) and Tagliabue et al. (2007): A dominant-hand advantage of the right-handed participants produced a larger Simon effect for stimuli at the right location than for stimuli at the left location, and this relation was not evident for left-handed participants. This lack of reversal in left-handed participants was likely due to hand dominance not being as strong for the left-handers as for the right-handers, and that our using a less strict criterion $( \pm 40-100)$ for categorizing participants' handedness than Rubichi and Nicoletti used ( $\pm 80-100)$. An analysis of only those left-handed participants with a handedness score of -80 to $100(\mathrm{~N}=16)$ showed the expected reversal of the asymmetry: The Simon effect was significant for responses to left stimuli $(\Delta M=17 \mathrm{~ms}, p=.011)$ but not right stimuli $(\Delta M=6$ ms, $p=.491)$. The Simon effect decreased across the RT distribution for both right- and left-handed persons, similar to Rubichi and Nicoletti's Experiment 2 and most visual Simon effect studies (Proctor et al., 2011). The dominanthand advantage for right-handed participants increased significantly in Rubichi and Nicoletti's Experiment 2 and numerically in our Experiment 1 . The slightly larger Simon effect and 
a) Spatial-Manual

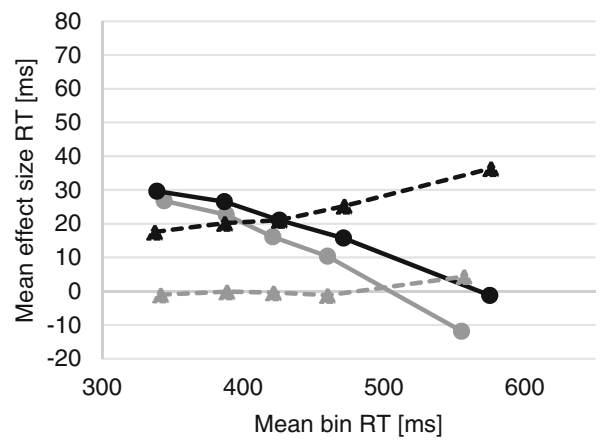

C) Spatial-Vocal

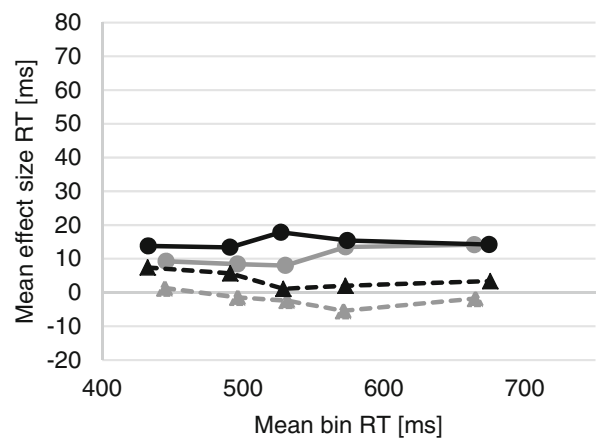

b) Verbal-Manual

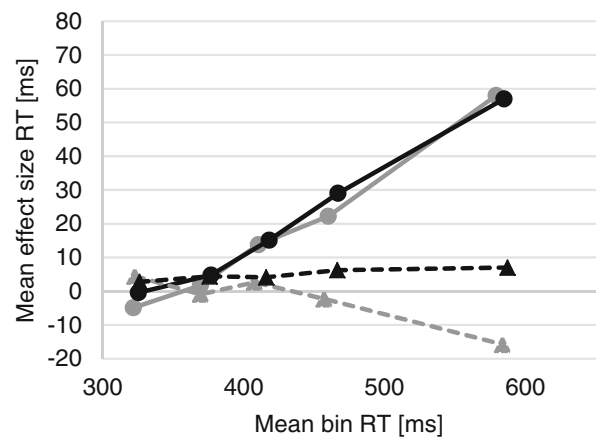

d) Verbal-Vocal

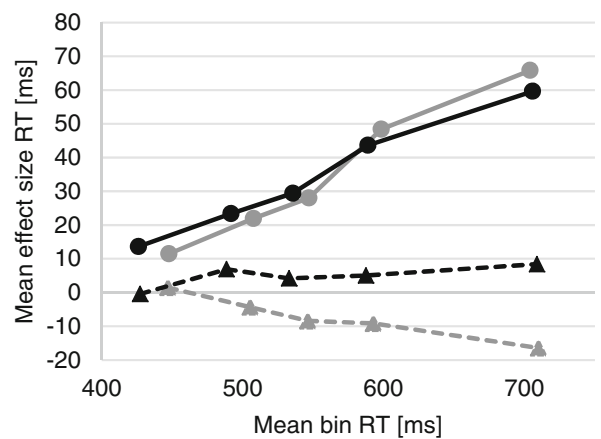

$\longrightarrow$ left-handed SE $\longrightarrow$ right-handed SE $\quad \longrightarrow---$ left-handed DHA $\quad--\llcorner--$ right-handed DHA

Fig. 2 Experiment 1: Reaction time (RT) distribution of the Simon effect (SE; continuous lines) and the dominant-hand advantage (DHA; dashed lines) for the spatial-manual condition. Grey lines indicate left-handed participants, whereas black lines indicate right-handed participants

dominant-hand advantage in their Experiment 2 was probably due to the fact that participants performed with their hands crossed.

Although the verbal-manual, spatial-vocal, and verbal-vocal conditions all yielded Simon effects, none of the conditions showed evidence of an asymmetry, for either right- or left-handed participants, based on whether the stimulus was left or right. This result implies that it is the unique combination of spatial stimuli and manual responses, both of which vary in left and right physical locations, that produces the Simon effect asymmetry reported by Rubichi and Nicoletti (2006). Assuming that preparation of the left and right hands induces a bias to attend to stimuli on the dominant-hand side, this outcome is in agreement with the attention-shift hypothesis favored by Rubichi and Nicoletti, Tagliabue et al. (2007), and Spironelli et al. (2009): Only the spatialmanual condition has the combination of hand dominance and difference in sides to which attention can be shifted. The problem with that account, though, is that our result pattern implies that the dominant-hand advantage is a main effect that speeds responding overall, rather than a direct contributor to the Simon effect.

\section{Experiment 2}

The unique property of Experiment 1's spatial-manual condition, which showed the dominant-hand advantage, is that it allows spatial matching of visual stimulus and response locations. Thus, in Experiment 2, in which we tested only righthanded participants, we eliminated visibility of the response locations (and responding hands) for one group by having them perform the spatial-manual task with their hands and response keys occluded by a wooden box. Another group performed with their hands in view, as a complete replication of the spatial-manual condition from Experiment 1. In addition to providing a baseline, this replication group allowed us to make sure that the result pattern of Experiment 1, which suggested that handedness had only a main effect, was not a consequence of intermixing the spatial-manual task with the other three task conditions.

If the Simon effect asymmetry for left and right stimulus locations requires visibility of the responses, there should be no asymmetry when the response hands are occluded, and the results should look similar to those of the spatial-verbal condition in Experiment 1. If this asymmetry is equally evident when the hands are visible and when they are not, and 
response location exerts only a main effect, then the results would speak against a visual matching strategy of response locations to stimulus locations. Such a result would suggest that the Simon effect asymmetry in stimulus locations could be a kind of general motoric preparation of the dominant hand response that is triggered by spatial stimuli.

\section{Method}

Participants Forty-eight new right-handed undergraduate students from the same subject pool as in Experiment 1 were invited to participate based on their handedness. There were 24 participants (15 males) in the group that was able to see their hands, whereas another 24 (eight males) had their hands occluded during the experiment. The participants' mean age was 19.5 years $(S D=1.29)$ in the first group and 19.6 years $(S D=1.18)$ in the second group. The Edinburgh handedness questionnaire (Oldfield, 1971) revealed a mean score of 79.51 $(S D=19.99)$ for the visible hands group and 84.37 ( $S D=$ 16.77) for the occluded hands group. The groups did not differ significantly regarding the strength of their right-handedness $(p=.376)$.

Apparatus, stimuli, and procedure The apparatus and stimuli were the same as in the spatial-manual condition of Experiment 1, with the exception of a large wooden box (56 $\mathrm{cm}$ wide $\times 46 \mathrm{~cm}$ deep $\times 20.5 \mathrm{~cm}$ high), painted black, enclosed on all but the front side, into which participants in the occluded hands group placed their hands while performing the task. Hence, the experiment contained 12 practice trials, followed by 120 experimental trials with stimulus colors and locations randomly intermixed. Participants filled out the Edinburgh handedness inventory (Oldfield, 1971) and were told to respond as fast and accurately as possible to the color of each stimulus, as in Experiment 1. The response-key-tostimulus-color mapping was counterbalanced between participants. Half of the participants performed the task with the response box (and their hands) being under the wooden box, which was placed on the desk and below the display screen. They were first allowed to lower their head to see their hands and the response box, which was placed inside the wooden box, and then after putting their fingers on the two outer keys of the response box they were told not to look at their hands while performing the task. The other half of participants could see their hands and the response box, as in Experiment 1. The experimenter stayed in the room, as in Experiment 1, but did not enter responses.

\section{Results}

ANOVAs were performed with stimulus location (left stimulus vs. right stimulus) and response location (left location vs. right location) as within-subject variables, and hand visibility (visible vs. occluded) as a between-subjects variable (see Fig. 3).

Reaction times A main effect of response location was found, $F(1,46)=10.06, p=.003, \eta^{2}=.18$, which indicates faster responses of the dominant right hand than of the left hand ( $\Delta M=10 \mathrm{~ms})$. Stimulus location and response location interacted, $F(1,46)=21.74, p<.001 \eta^{2}=.32$, producing a Simon effect (see Fig. 3). Orthogonal contrasts showed that for the left stimulus, the Simon effect was only marginally significant $(\Delta M=11 \mathrm{~ms}, p=.064)$, whereas for the right stimulus the Simon effect was more clearly evident $(M=$ $29 \mathrm{~ms}, p<.001)$. This time, the non-corresponding trials differed significantly between stimulus locations $(\Delta M=$ $8 \mathrm{~ms}, p=.001)$, but the corresponding trials did not $(\Delta M=13 \mathrm{~ms}, p=.093)$.

No other interaction was significant. Most importantly, the data show that seeing their hands made no difference between the hand visibility groups, $F<1.0$ (see Fig. 3 ).

Percent errors For PEs (see Fig. 3), the ANOVA showed only a significant stimulus location $\times$ response location interaction, $F(1,52)=9.78, p=.003, \eta^{2}=.04$. Similar to the RT data, the Simon effect for the left stimulus side $(\Delta M=$ $1.0 \%, p=.051)$ was smaller than that for the rightand dominant-hand stimulus side $(\Delta M=1.4 \%, p=.018)$. Neither corresponding nor non-corresponding trials differed in their error rates between stimulus sides $(\Delta M=0.3 \%$, $p=.520 ; \Delta M=0.02 \%, p=.978$ ). The group factor of hand visibility did not interact with the Simon effect, $F(1,46)=0.2$, $p=.627, \eta^{2}=.01$.

Distribution analyses A distribution analysis was performed as in Experiment 1 (see Fig. 4). ANOVAs of the RT data, with factors of Simon effect, or dominant-hand advantage, bin, and the between-subjects factor of hand visibility. They showed the expected main effect of bin and the Simon effect. The latter decreased significantly across bins, $F(1.7,76.1)=6.91$, $p=.003, \eta^{2}=.13$, but this decreasing pattern did not interact with hand visibility $(F=1.28, p=.280)$. In PE, a main effect of bin was evident, as was the Simon effect, which decreased significantly across bins, $F(2.7,122.3)=10.43, p<.001$, $\eta^{2}=.19$. Hand visibility had no influence here, too $(F<1.0$, $p=.432)$. The dominant-hand advantage was only present in the RT distribution, $F(1,46)=9.64, p=.003, \eta^{2}=.17$, and it did not vary between bins $(F=1.35, p=.263)$, nor was it influenced by hand visibility $(F<1.0, p=.406)$.

\section{Discussion}

The results with the hands visible replicated those obtained for the spatial-manual condition in Experiment 1's right-handed group: There was a main effect of response location, and this 


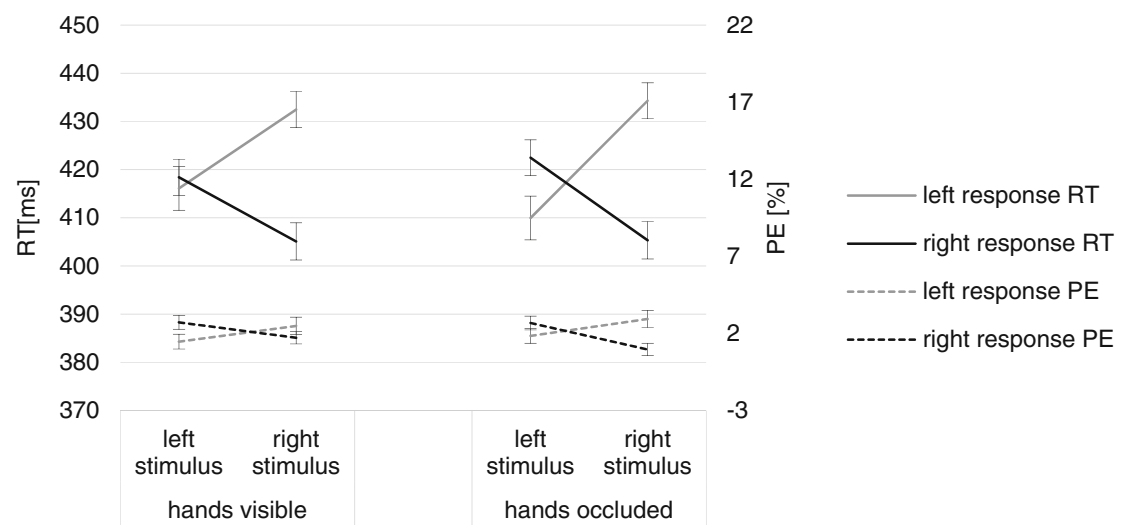

Fig. 3 Experiment 2: Mean reaction time (RT) and percent error (PE) values and Cousineau-Morey confidence intervals (controlling for between-subjects variability; Morey, 2008), as functions of stimulus location (left stimulus vs. right stimulus), response location (left response vs. right response), and hand visibility (hands visible vs. hands invisible) for the spatial stimuli and manual responses factor interacted with stimulus location, indicating the Simon effect. As a consequence of the advantage for the dominant right hand, the Simon effect was larger for the right stimulus location than for the left stimulus location. This relation was evident regardless of whether the hands were visible or occluded, indicating that sight of the hands is not necessary for a dominant-hand advantage to occur. This outcome speaks in favor of a spatial matching rather than of a visual matching of stimuli and responses, and such a motoric preparation of the dominant hand seems to be stronger when spatial stimuli are present.

Wallace $(1971,1972)$ conducted conditions similar to these across three experiments in which right-handed participants pressed left and right keys to indicate whether a stimulus was a circle or a square. We focus only on conditions for which the hands were uncrossed, as in the present study. In his 1971 experiment, six participants performed with full vision of their hands and showed a Simon effect asymmetry: 35ms effect for left stimulus and 73-ms effect for right stimulus. Six different participants in Experiment 1 of his 1972 study showed similar effects of 25 and $73 \mathrm{~ms}$ for left and right stimuli. In another condition of that experiment, six participants performed wearing a halter that prevented seeing the hands. These participants showed no asymmetry, with the Simon effects being $55 \mathrm{~ms}$ and $48 \mathrm{~ms}$ for the left and right stimuli, respectively. Experiment 2 differed from the halter condition of Experiment 1 in that the participants had their hands placed passively on the keys by the experimenter. The four participants who performed with uncrossed hands again showed no Simon effect asymmetry, with the left stimulus Simon effect being $68 \mathrm{~ms}$ and the right stimulus one being $65 \mathrm{~ms}$.

So, collapsing across experiments, Wallace's (1971, 1972) results with 12 participants who could see their hands and ten who could not, differ from our results: The asymmetry was evident only for the participants whose hands were visible. Disregarding the small number of participants from Wallace's experiments, there are several differences between his experiments and ours that could be the source of the apparently discrepant results: Stimulus discrimination was of shapes instead of colors, stimuli in vertical top and bottom locations were intermixed with the stimuli in left and right positions, and the monitor placement was higher. Yet, there is no reason why these variables should interact with visibility

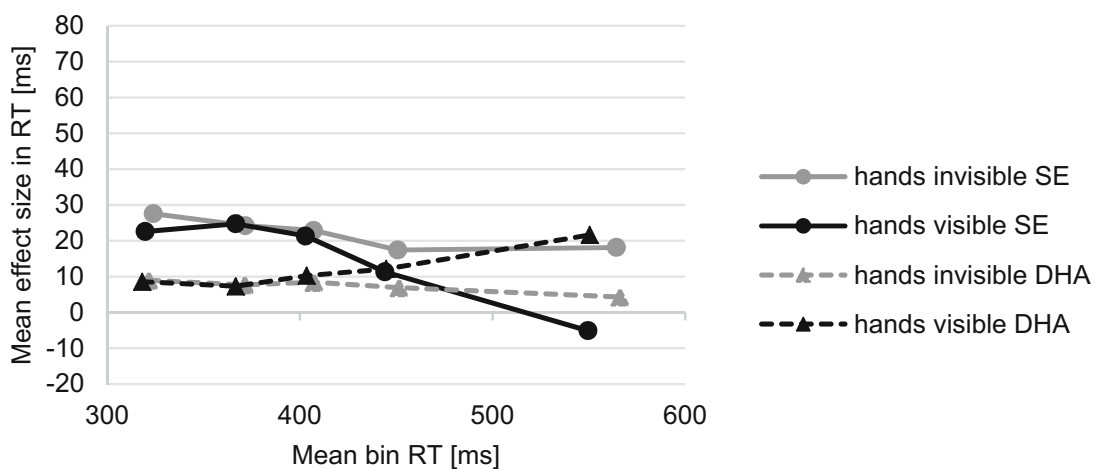

Fig. 4 Experiment 2: Reaction time (RT) distribution of the Simon effect (SE; continuous lines) and the right-hand advantage (DHA; dashed lines) for participants with their response hands visible (grey lines) or response hands occluded (black lines). The data represent spatial stimuli and manual responses in which only right-handed participants were tested 
of the hands. The most likely reason is that the participants were never able to see their response keys or hands from the time they entered the darkened experiment room in Wallace's experiment, whereas in ours the participants were able to see the response box and hands prior to positioning themselves to start the practice trials. Hence, the preliminary visibility of the hands and keys may be sufficient for participants to form a spatial representation for the left and right responses.

\section{General discussion}

Our experiments aimed at examining the earlier findings of asymmetric Simon effects for left and right stimuli in the visual Simon task more closely. Rubichi and Nicoletti (2006) and Tagliabue et al. (2007) showed that the Simon effect is larger and significant for the stimulus on the dominant hand side, compared to that for the stimulus on the subordinate hand side. The authors explained this asymmetry by a bias in attention shifting towards the side where the dominant hand is currently operating. They also noted that current models explaining the Simon effect lack a mechanism to explain the asymmetry on the stimulus-location level.

The present study's aim therefore was to conduct some additional conditions that enabled us to narrow down the range of situations under which this asymmetry can be found and to determine whether the biased attention-shift explanation holds up. We evaluated the assumption that handedness in the form of an RT advantage of the dominant hand is responsible for the spatial-manual Simon effect asymmetry by having participants make vocal, location-word responses. We also used verbal location-word stimuli to assess whether the asymmetry only occurs when participants have to shift attention to a left or right stimulus location, as for exogenous spatial locations.

In Experiment 1, four different S-R mode conditions were tested: (a) spatial-manual, (b) verbal-manual, (c) spatial-vocal, and (d) verbal-vocal. The results of the spatial-manual condition for the most part replicated those of Rubichi and Nicoletti (2006), with an exception being that the left-hand dominant group did not show a significant reversal of the stimulus location-based Simon effect asymmetry shown by the righthanded group. As noted, this lack of reversal was probably due to a less strict criterion for categorizing participants' handedness than Rubichi and Nicoletti used, as the strongest lefthanders showed the reversal.

Furthermore, following the data analyses of Tagliabue et al. (2007), the results show that the asymmetry in the Simon effect, when it is analyzed with respect to correspondence with stimulus locations (as in Rubichi \& Nicoletti, 2006), is represented by a significant interaction of handedness and response location when it is analyzed with respect to response and stimulus locations. Hence, indicative of the asymmetry, a large dominant-hand advantage in right-handed participants was found.

There was a minor deviation of the present findings from previous ones: The difference between the corresponding trials for the two stimulus locations was not larger than the difference between the non-corresponding trials, as it looked like in some of the prior studies (e.g., Rubichi \& Nicoletti's, 2006, Experiment 1 but not their Experiment 2; Valle-Inclán's, 1996, Experiment 1). The reason seems to be the absence of a stimulus location bias in our data, whereas in the studies finding a difference in corresponding trials, a bias of stimulus location towards the dominant hand side was found. Such a slight tendency for a stimulus location bias when looking at the main effect might produce a significant Simon effect asymmetry in the orthogonal comparisons of the Simon effect interaction components (although we have to be cautious interpreting main effects when an interaction is present, too). With both a stimulus and a response location main effect, which are both biased towards the dominant hand, a corresponding dominant-hand response is the fastest response of a right-handed participant, because stimulus and response location bias are both in favor of this condition. For a corresponding response to a left stimulus, the response location and stimulus location bias both prolong the RT. For noncorresponding trials, in contrast, stimulus and response location bias counter each other. This means that the two factors increase the difference between corresponding trials but decrease the difference between non-corresponding trials. When reviewing several studies on the Simon effect that plot their data in a similar way and find similar Simon effect asymmetries, the most consistent finding is a dominant-hand advantage (see e.g., Proctor \& Wang, 1997; Rubichi \& Nicoletti, 2006; Schroeder-Heister, Heister, \& Ehrenstein, 1988; Simon, Sly, \& Vilapakkam, 1981; Spironelli et al., 2009; Tagliabue et al., 2007; Valle-Inclán, 1996). The stimulus location bias varies in its direction a lot between studies and is only present in some of the reviewed ones (e.g., Rubichi \& Nicoletti, 2006; Spironelli et al., 2009; Tagliabue et al., 2007; Wallace, 1972). Again, it was not evident in either of our experiments. Despite that, the critical result of unequally sized Simon effects with respect to stimulus locations was obtained, which is why we focus on the dominant-hand advantage here, as it should be the main cause of the asymmetry in our data.

As response location (and therefore the dominant-hand advantage) is a part of the Simon effect interaction, there was a need to examine whether response location interacts with the Simon effect or just has additive effects on the latter. Therefore, RT distribution functions were calculated on the data. The Simon effect function, obtained in the spatialmanual condition, showed a decreasing trend, which is typically attributed to dissipation of the physical stimulus location's automatic response activation (see e.g., De Jong et al., 
1994; Töbel et al., 2014). A dominant-hand advantage that is a part of the actual Simon effect in the spatial-manual condition should show the decreasing trend, too. However, the opposite was found in the present data, as well as in those of Rubichi and Nicoletti (2006). More precisely, in the present data a constant dominant-hand RT advantage was found, and in the study of Rubichi and Nicoletti even an increasing pattern although the latter must be regarded cautiously, because an increase in the fifth "outlier" could be an artifact. In both their and our experiments a dominant-hand RT advantage was already present in the first RT bin (although only numerically with $11 \mathrm{~ms}$ in Rubichi and Nicoletti, 2006), which means that some general pre-activation of the dominant hand must have been present that persisted also in longer RTs. One could infer from these data that the Simon effect asymmetry is not "real," in the sense of reflecting an influence on the mechanism responsible for the Simon effect, as they appear at different stages in the response process.

This latter interpretation is consistent with the literature on the Simon effect, as compared to the idea of Rubichi and Nicoletti (2006). A lot of studies on the Simon effect showed that correspondence relations usually are determined by exterior spatial relations and that anatomical factors play a negligible role. Experiments like that of Wallace (1971), in which participants responded with their hands crossed, did not show a reversal of the Simon effect: the Simon effect still followed stimulus and response key locations and not the location of the anatomical hand. Therefore, Rubichi and Nicoletti's idea of an attentional bias centered on the dominant hand that diminishes the correspondence effect on the non-dominant hand's stimulus side seems to go against such earlier findings. With the present results in mind, which indicate additive effects of a dominant-hand RT advantage, the latter interpretation seems even more questionable to us.

Still, the origin of the dominant hand advantage is unclear. Namely, apart from the right-handed participants in the spatial-manual condition, no other experimental condition showed the asymmetry in Experiment 1. The absence of asymmetry in the verbal-vocal condition was expected because neither handedness nor an attention shift to a stimulus location is a factor. More importantly, there was no locationword Simon effect asymmetry in the verbal-manual condition, which suggests that it is neither a semantic code nor name code generated by the stimulus, nor the intention of giving a left or right manual response, that produces the asymmetry in the spatial-manual condition. Moreover, it indicates that the RT advantage of the dominant hand is not a simple motor effect - although there is empirical evidence for physiological factors like lower motor thresholds for dominant-hand responses (see, e.g., Adam, De Luca, \& Erim, 1998). The absence of an asymmetry in vocalization onsets of the spatialvocal condition provides evidence that participants were not coding responses with respect to their hands or physical locations when responding with the spoken words "left" and "right." It is possible that a motoric speech measure, such as the mouth opening being wider on the right than left side (Hausmann et al., 1998; Wolf \& Goodale, 1987), would show indication of a Simon effect asymmetry, but this is unlikely since it would require a complex interaction between the vocal responses "left" and "right" and the left and right stimuli. Therefore, again, we think that the underlying RT advantage of the dominant hand in the spatial-manual task cannot be produced by a general attentional bias that favors one spatial stimulus location over the other. The interplay of factors producing the dominant-hand advantage seems to be more complex than proposed by Rubichi and Nicoletti (2006).

As our results show, the dominant-hand advantage, and thereby asymmetric Simon effects for left and right stimulus locations, seems to depend on the physical spatial task environment. Hence, spatial-motor response preparation could be at the origin of the effect. Namely, the exogenous nature of physical spatial stimuli and spatial response locations could be critical for the asymmetries. Exogenous attention attraction is said to happen automatically and is triggered by the saliency of the physical characteristics of a stimulus. The latter can be an abrupt lateral onset of the stimulus (see, e.g., Janczyk, Pfister, Wallmeier, \& Kunde, 2014; Jonides, 1981), as in the present experiment, in contrast to endogenous stimuli that need semantic processing to trigger a response. Our data suggest that the dominant-hand advantage is produced by some kind of spatial-motoric response preparation. Such general preparation for a dominant-hand response might only be present when the task requires motor-interaction with a spatial environment, which was the case in the spatial-manual condition only. Moreover, the preparation must be very basic and does not influence the response-selection stage. If it influenced response selection, the dominant-hand advantage would also be present in error rates, like in the Simon effect, but this was not the case.

Still, these arguments cannot rule out the possibility entirely that an attentional dominant-hand centered bias, like that proposed by Rubichi and Nicoletti (2006), is responsible for the effect. One could infer from Rubichi and Nicoletti's experiment with crossed hand positions that the dominant hand needs to be actively involved in the task to produce the asymmetry through an attention bias. Nevertheless, a more economical explanation for the absence of a dominant-hand advantage in the spatial-vocal condition might be provided by the referential coding account (Hommel, 1993b), which assumes that spatial relations in a task are defined by taskspecific frames of reference. As stimuli and responses use similar left-right spatial frames of reference in the spatialmanual condition, interference on this level is quite plausible, whereas for spatial stimuli and verbal responses the reference frames differ, and therefore physical spatial sides will not be a factor. 
This explanation in terms of different reference frames implies that the visibility of the response locations may be a critical factor for selecting those frames and therefore also for producing a dominant-hand advantage. These thoughts led us to conduct Experiment 2, in which two groups of right-handed participants were compared in the spatialmanual condition. One group was allowed to see their hands and the response box during the task and the other was not able to do so. The results did not show a difference between the two groups. Still, to our understanding the explanation of the dominant-hand advantage as dependent on task-specific frames of reference does not necessarily have to be discarded: We cannot answer the question, whether initially being able to see the response box and placement of one's hands on it is essential. Results of Wallace (1972) seem to show no Simon effect asymmetry when the experimental room was darkened to eliminate visual cues from the time a participant entered the room. This issue would be an interesting one to examine in future experiments.

Despite the difficulties in finding a further falsifiable hypothesis about the origin of the dominant-hand advantage, and by that the Simon effect asymmetries, the present study revealed some details that have considerable practical implications for future research on the Simon effect. The recommendation to avoid calculating overall Simon effects rather than analyzing the data by response location does not need to be heeded. Namely, the data show that overall Simon effects should not be affected by an underlying dominant-hand advantage that yields the asymmetries within the Simon effect interaction: The interaction of the overall Simon effect and handedness was not significant, although in the spatialmanual task, the right-handed participants but not the lefthanded ones showed the asymmetries.

The reason why Rubichi and Nicoletti (2006) reached a different conclusion was that both their left- and righthanded participants did show an asymmetric Simon effect pattern when the effect was calculated based on correspondence with stimulus location. Therefore, they assumed that both handedness groups had equal sized although opposite asymmetries. In the overall Simon effect the opposite pattern is collapsed and, therefore, no difference in the overall Simon effect could be found anymore. Yet, the present findings indicate that when counterbalancing stimulus and response sides, asymmetric features that are independent from the correspondence connections in the Simon effect are averaged out.

This analysis means that the Simon effect is quite robust and will emerge independent from biases like the participant's handedness. In contrast to Tagliabue et al. (2007), we do not recommend to avoid calculating an overall Simon (correspondence) effect when analyzing Simon tasks. Instead, we advise sticking to the practice of evaluating overall Simon effects instead of calculating correspondence effects separately for each stimulus location or each response location, because the latter confound stimulus-identification or response factors with response selection, thus producing results that are prone to interpretation errors.

Author note We thank Jochen Müsseler, Julia Seibold's advisor at RWTH Aachen University, and the RWTH - PROMOS scholarship of the DAAD for their support of this research at Purdue University, and Bernhard Hommel and an anonymous reviewer for helpful comments on earlier versions of the manuscript.

\section{References}

Adam, A., De Luca, C. J., \& Erim, Z. (1998). Hand dominance and motor unit firing behavior. Journal of Neurophysiology, 80, 1373-1382. doi:10.1016/0006-8993(92)90625-J

Ansorge, U., \& Wühr, P. (2004). A response-discrimination account of the Simon effect. Journal of Experimental Psychology: Human Perception and Performance, 30, 365-377. doi:10.1037/00961523.30.2.365

De Jong, R., Liang, C. C., \& Lauber, E. (1994). Conditional and unconditional automaticity: A dual-process model of effects of spatial stimulus-response correspondence. Journal of Experimental Psychology: Human Perception and Performance, 20, 731-750. doi:10.1037/0096-1523.20.4.731

Fitts, P. M., \& Deininger, R. L. (1954). S-R compatibility: Correspondence among paired elements within stimulus and response codes. Journal of Experimental Psychology, 48, 483-492. doi: $10.1037 / \mathrm{h} 0054967$

Hausmann, M., Behrendt-Körbitz, S., Kautz, H., Lamm, C., Radelt, F., \& Güntürkün, O. (1998). Sex differences in oral asymmetries during word repetition. Neuropsychologia, 36, 1397-1402. doi:10.1016/ S0028-3932(98)00027-X

Hedge, A., \& Marsh, N. W. (1975). The effect of irrelevant spatial correspondences on two-choice response-time. Acta Psychologica, 39, 427-439. doi:10.1016/0001-6918(75)90041-4

Hommel, B. (1993a). Inverting the Simon effect by intention Determinants of direction and extent of effects of irrelevant spatial information. Psychological Research, 55, 270-279. doi:10.1007/ BF00419687

Hommel, B. (1993b). The role of attention for the Simon effect. Psychological Research, 55, 208-222. doi:10.1007/BF00419608

Hommel, B. (2011). The Simon effect as tool and heuristic. Acta Psychologica, 136, 189-202. doi:10.1016/j.actpsy.2010.04.011

Hommel, B., Müsseler, J., Aschersleben, G., \& Prinz, W. (2001). The theory of event coding (TEC): A framework for perception and action planning. Behavioral and Brain Sciences, 24, 849-878. doi: 10.1017/S0140525X01000103. discussion 878-937.

Janczyk, M., Pfister, R., Wallmeier, G., \& Kunde, W. (2014). Exceptions to the PRP effect? A comparison of prepared and unconditioned reflexes. Journal of Experimental Psychology: Learning, Memory, and Cognition, 40, 776-786. doi:10.1037/a0035548

Jonides, J. (1981). Voluntary versus automatic control over the mind's eye's movement. In J. B. Long \& A. D. Baddeley (Eds.), Attention and performance $I X$ (pp. 187-203). Hillsdale: Lawrence Erlbaum. doi:10.1037/0096-1523.29.5.835

Kornblum, S., Hasbroucq, T., \& Osman, A. (1990). Dimensional overlap: Cognitive basis for stimulus-response compatibility-a model and taxonomy. Psychological Review, 97, 253-270. doi:10.1037/0033295X.97.2.253

Lu, C., \& Proctor, R. W. (1995). The influence of irrelevant location information on performance: A review of the Simon and spatial 
Stroop effects. Psychonomic Bulletin \& Review, 2, 174-207. doi:10. 3758/BF03210959

Morey, R. D. (2008). Confidence intervals from normalized data: A correction to Cousineau (2005). Tutorial in Quantitative Methods for Psychology, 4, 61-64. doi:10.3758/s13414-012-0291-2

Nicoletti, R., Anzola, G. P., Luppino, G., Rizzolatti, G., \& Umiltà, C. (1982). Spatial compatibility effects on the same side of the body midline. Journal of Experimental Psychology: Human Perception and Performance, 8, 664-673. doi:10.1037/0096-1523.8.5.664

Nicoletti, R., \& Umiltà, C. (1994). Attention shifts produce spatial stimulus codes. Psychological Research, 56, 144-150. doi:10.1007/ BF00419701

Nicoletti, R., Umiltà, C., \& Mapelli, D. (1992). Spatial representations of words and nonwords. Cortex, 28, 163-174. doi:10.1016/S00109452(13)80045-7

Oldfield, R. C. (1971). The assessment and analysis of handedness: The Edinburgh inventory. Neuropsychologia, 9, 97-113. doi:10.1016/ 0028-3932(71)90067-4

Proctor, R. W., Miles, J. D., \& Baroni, G. (2011). Reaction time distribution analysis of spatial correspondence effects. Psychonomic Bulletin \& Review, 18, 242-266. doi:10.3758/s13423-011-0053-5

Proctor, R. W., \& Vu, K. P. L. (2006). Stimulus-response compatibility principle: Data, theory, and application. Boca Raton: CRC Press. doi: $10.1201 / 9780203022795$

Proctor, R. W., \& Wang, H. (1997). Enhancement of the Simon effect by response-location precues: Evaluation of the stimulus-identification account. Acta Psychologica, 95, 279-298. doi:10.1016/S00016918(96)00044-3

Rabbitt, P. (1978). Hand dominance, attention, and the choice between responses. Quarterly Journal of Experimental Psychology, 30, 407416. doi:10.1080/00335557843000016

Ratcliff, R. (1979). Group reaction time distributions and an analysis of distribution statistics. Psychological Bulletin, 86, 446-461. doi:10. 1037/0033-2909.86.3.446

Ridderinkhof, K. R. (2002). Activation and suppression in conflict tasks: Empirical clarification through distributional analyses. In W. Prinz \& B. Hommel (Eds.), Common mechanisms in perception and action: Attention and performance XIX (pp. 494-519). Oxford: Oxford University Press.

Rubichi, S., \& Nicoletti, R. (2006). The Simon effect and handedness: Evidence for a dominant-hand attentional bias in spatial coding. Perception \& Psychophysics, 68, 1059-1069. doi:10.3758/ BF03193709

Schroeder-Heister, P., Heister, G., \& Ehrenstein, W. H. (1988). Spatial SR compatibility under head tilt. Acta Psychologica, 69, 35-44. doi: 10.1016/0001-6918(88)90028-5

Simon, J. R. (1990). The effects of an irrelevant directional cue on human information processing. In R. W. Proctor \& T. G. Reeve (Eds.), Stimulus-response compatibility: An integrated perspective (pp. 31-86). Amsterdam: North-Holland. doi:10.1016/S0166-4115(08) 61218-2
Simon, J. R., Sly, P. E., \& Vilapakkam, S. (1981). Effect of compatibility of S-R mapping on reactions toward the stimulus source. Acta Psychologica, 47, 63-81. doi:10.1016/0001-6918(81)90039-1

Simon, J. R., \& Small, A. M. (1969). Processing auditory information: Interference from an irrelevant cue. Journal of Applied Psychology, 53, 433-435. doi:10.1037/h0028034

Spironelli, C., Tagliabue, M., \& Umiltà, C. (2009). Response selection and attention orienting a computational model of Simon effect asymmetries. Experimental Psychology, 56, 274-282. doi:10.1027/ 1618-3169.56.4.274

Tagliabue, M., Vidotto, G., Umiltà, C., Altoè, G., Treccani, B., \& Spera, P. (2007). The measurement of left-right asymmetries in the Simon effect: A fine-grained analysis. Behavior Research Methods, 39, 50 61. doi:10.3758/BF03192843

Töbel, L., Hübner, R., \& Stürmer, B. (2014). Suppression of irrelevant activation in the horizontal and vertical Simon task differs quantitatively not qualitatively. Acta Psychologica, 152, 47-55. doi:10. 1016/j.actpsy.2014.07.007

Townsend, J. T., \& Ashby, F. G. (1978). Methods of modeling capacity in simple processing systems. In J. Castellan \& F. Restle (Eds.), Cognitive theory (3rd ed., pp. 200-239). Hillsdale: Lawrence Erlbaum.

Umiltà, C., \& Nicoletti, R. (1985). Attention and coding effects in S-R compatibility due to irrelevant spatial cues. In M. I. Posner \& O. S. Marin (Eds.), Attention and Performance XI (pp. 457-471). Hillsdale: Lawrence Erlbaum.

Valle-Inclán, F. (1996). The locus of interference in the Simon effect: An ERP study. Biological Psychology, 43, 147-162. doi:10.1016/03010511(95)05181-3

Valle-Inclán, F., Sohn, F., \& Redondo, M. (2008). Spatial compatibility between stimulated eye and response location. Psychophysiology, 45, 279-285. doi:10.1111/j.1469-8986.2007.00616.x

Wallace, R. J. (1971). S-R compatibility and the idea of a response code. Journal of Experimental Psychology, 88, 354-360. doi:10.1037/ h0030892

Wallace, R. J. (1972). Spatiel S-R compatibility effects involving kinesthetic cues. Journal of Experimental Psychology, 93, 163-168. doi: $10.1037 / \mathrm{h} 0032462$

Wolf, M. E., \& Goodale, M. A. (1987). Oral asymmetries during verbal and non-verbal movements of the mouth. Neuropsychologia, 25, 375-396. doi:10.1016/0028-3932(87)90026-1

Wühr, P. (2006). The Simon effect in vocal responses. Acta Psychologica, 121, 210-226. doi:10.1016/j.actpsy.2004.12.003

Xiong, A., \& Proctor, R. W. (2015). Decreasing auditory Simon effects across reaction time distributions. Journal of Experimental Psychology: Human Perception and Performance. doi:10.1037/ xhp0000117

Yamaguchi, M., \& Proctor, R. W. (2012). Multidimensional vector model of stimulus-response compatibility. Psychological Review, 119, 272-303. doi:10.1037/a0026620 